\title{
Self-focus and estimation of heart rate following physical exertion
}

\author{
ROD GILLIS and CHARLES S. CARVER \\ University of Miami, Coral Gables, Florida 33124
}

\begin{abstract}
A study investigated the hypothesis that highly self-attentive persons would be more accurate in estimating their heart rates than would less self-attentive persons. Subjects' heart rates were elevated by approximately $60 \mathrm{bpm}$ by physical exercise. Subjects then estimated their heart rates at 30-sec intervals, as they returned to their preexercise baselines. Estimates were compared with subjects' actual heart rates. The study failed to yield support for the hypothesis being tested. Discussion centers on possible reasons for this failure.
\end{abstract}

Recent research suggests that when a person is experiencing emotional arousal, an increase in selfdirected attention leads to increased awareness of that emotional state (e.g., Carver, Blaney, \& Scheier, 1979; Scheier \& Carver, 1977). This has occurred whether self-focus was experimentally manipulated or subjects were divided according to their chronic dispositions to be self-attentive. As an example, Scheier and Carver (1977) presented subjects with photographic slides (either repulsive or sexually arousing) that were assumed to produce some autonomic changes. It was expected that all subjects would be aware of these changes to some degree but that the changes would be most salient among highly self-focused subjects. As predicted, selfattentive persons rated the attractive slides more favorably and the repulsive slides more negatively than did less self-attentive subjects.

Further research indicates that self-focus leads to enhanced awareness of the absence of arousal, as well as the presence of arousal. In one experiment (Gibbons, Carver, Scheier, \& Hormuth, 1979), subjects who were led to expect arousal from a nonarousing placebo were less likely to report arousal when self-attention was increased by mirror presence than when it was not. This reasoning has even been extended to the experience of taste (Scheier, Carver, \& Gibbons, 1979, Experiment 2), subjects high in dispositional self-consciousness being more accurate in reporting a flavor intensity in the face of misleading information than less selfconscious subjects. These studies, taken together with those of Scheier and Carver (1977) suggest that selfattention enhances one's awareness of a wide variety of veridical internal states.

Scheier and Carver (1977) assumed that their experimental stimuli had induced some autonomic arousal in their subjects. Similarly, Gibbons et al. (1979)

This study was conducted as a Master's thesis by the first author under the supervision of the second author. Requests for reprints should be directed to Rod Gillis, Department of Psychology, P.O. Box 248185, University of Miami, Coral Gables, Florida 33124. assumed that their subjects were not aroused beyond their normal resting levels. However, no measurements of actual physiological state were made in any of those studies. The present study was an attempt to extend those findings by assessing the degree to which subjects were aware of their actual physiological states, in one specific modality: heart rate. Rather than examine emotional arousal, however, this study focused on heart rate variations produced by physical exertion.

\section{METHOD}

Subjects were 28 men and 28 women from the University of Miami subject pool. Subjects were divided into high and low self-consciousness groups (median split) on the basis of their scores on the private subscale ${ }^{1}$ of the Self-Consciousness Scale (Fenigstein, Scheier, \& Buss, 1975), which had been administered several weeks earlier. Fourteen of each sex were high in self-consciousness, and 14 were low. Each group was further divided into mirror and no-mirror conditions (described more fully below).

\section{Procedure}

Subjects were tested individually. Upon arrival, each subject was met by the experimenter and brought into the laboratory. While preparing and placing heart rate electrodes, the experimenter described the study's procedures. Once the electrodes were in place, and the subject's resting heart rate had been clearly established, the subject was asked to get on the exercise bicycle and to pedal rapidly for 1 or 2 min until his or her heart rate was sufficiently elevated. At that time, the subject was asked to return to the original seat.

The subject then saw a series of slides projected onto a rear-projection screen. The subject was to look at each slide carefully, so that he or she would be able to answer questions about the slides after all had been presented. The implication of these instructions was that the purpose of the experiment was to study the effects of physical exertion on memory.

The subjects were also asked to provide estimates of their own heart rates during the interval following each slide (i.e., every $30 \mathrm{sec}$ ). Subjects were to do this according to a hypothetical scale that ranged from 0 to 10 . Zero, they were told, represented their resting level; 10 represented the way their heart rate would feel to them as soon as they stepped off the bicycle. A visual scale with these endpoints clearly labeled was provided as an aid.

Experimental manipulation of self-attention. In addition to assessing the effect of chronic self-consciousness, self-focus 
was experimentally varied among subjects by the presence of a mirror. The mirror was introduced into the situation unobtrusively, as follows. The slides were rear-projected onto a small $(.5 \times .75 \mathrm{~m})$ screen, which stood a few feet in front of the subject. For half of the subjects, the screen was an ordinary nonreflective rear-projection screen (no-mirror condition). For the remainder of the subjects, the screen was made of a $50 \%$ reflective, $50 \%$ transmission glass (cf. Scheier \& Carver, 1977). Each subject facing this glass was able to view himself or herself in the intervals between slide presentations (mirror condition).

In addition to serving as a vehicle for manipulation of selfattention, the presentation of the slides was intended to serve a second purpose. Because of the possibility that all subjects might be at a psychological "ceiling" of self-attention, the slides (chosen as being somewhat complex in composition) were introduced as a distractor. Thus self-focus should have been somewhat reduced among all subjects. For subjects in the mirror condition, however, there was a stimulus that reminded subjects of themselves.

\section{Treatment of the Data}

An averaging procedure was established to arrive at a resting level for each subject. Ten heart beats were averaged from each of two periods, beginning $30 \mathrm{sec}$ and $60 \mathrm{sec}$ prior to the exercise task. The overall average of these data points was defined as the subject's base rate.

The highest rate obtained by the individual, invariably occurring at the moment the subject stopped pedaling on the bicycle, was simply read as that single point on the graph.

Ten actual heart rate values were computed during the period following the exercise. Each corresponded to one of the estimates made by subjects at $30-\mathrm{sec}$ intervals. Each actual heart rate value was obtained by averaging the six heart beats immediately prior to the event mark that indicated the time at which the corresponding estimate was made. Estimated heart rates, of course, had been obtained directly from the subject.

Note that actual heart rates during this period were recorded in terms of beats per minute (bpm), whereas heart rate estimates, on the other hand, were recorded on a scale of 0 to 10 . In order to make these two indices comparable, raw heart rate values during the postexercise period were standardized on a scale of 0 to 10 . These values were compared with the subject's heart rate estimates (see below).

\section{RESULTS}

\section{Subject Characteristics}

An analysis of variance of subjects' self-consciousness scores revealed no significant difference between groups other than the built-in main effect for self-consciousness. Analysis of subjects' resting heart rates prior to exertion revealed that men who were high in self-consciousness had marginally higher resting rates (mean $=84.26$ ) than men lower in self-consciousness $($ mean $=73.42)$ $(\mathrm{p}<.06)$.

It was intended that each subject's heart rate be increased by $60 \mathrm{bmp}$ during the exercise procedure. However, an analysis of subjects' range-of-elevation scores (peak heart rate minus base rate) indicated that the heart rates of men who were low in selfconsciousness had been elevated by greater amounts than the heart rates of men who were high in selfconsciousness $(\mathrm{p}<.01)$.

\section{Differences Between Actual and Estimated Rates}

Raw heart rate values during the postexercise period were converted to a 10-point standardized scale. Each estimate of heart rate then was subtracted from the corresponding standardized rate to provide a difference score. Ten difference scores were obtained for each subject in this manner. A repeated-measures analysis of variance was performed on the absolute values of these difference scores. This analysis yielded only one significant effect: a main effect for trials $[F(9,432)=39.12$, $\mathrm{p}<.001]$. As can be seen from Figure 1, this trials effect reflects increased accuracy of estimation in later trials. This effect reflects increased accuracy of estimation in later trials. This effect is most easily understood as reflecting the fact that both estimates and actual standardized heart rates eventually converged on 1 , the lowest value on the scale.

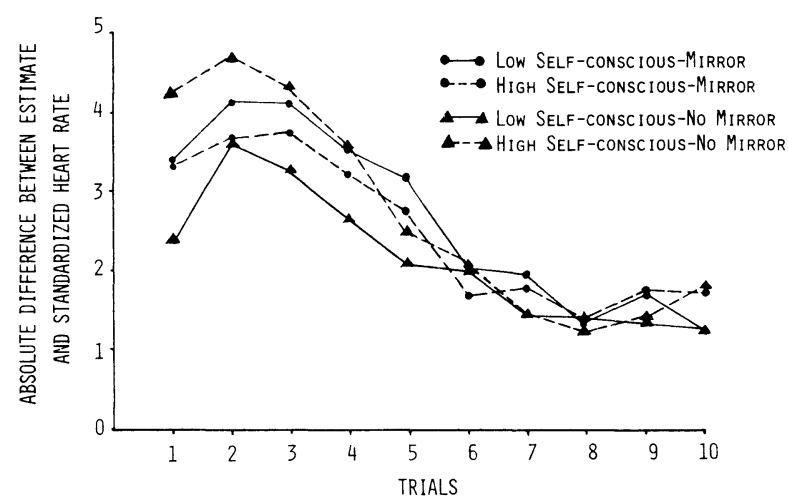

Figure 1. Absolute differences between estimates and standardized heart rates, over 1030 -sec intervals, among persons low and high in self-consciousness, with mirror present and mirror absent.

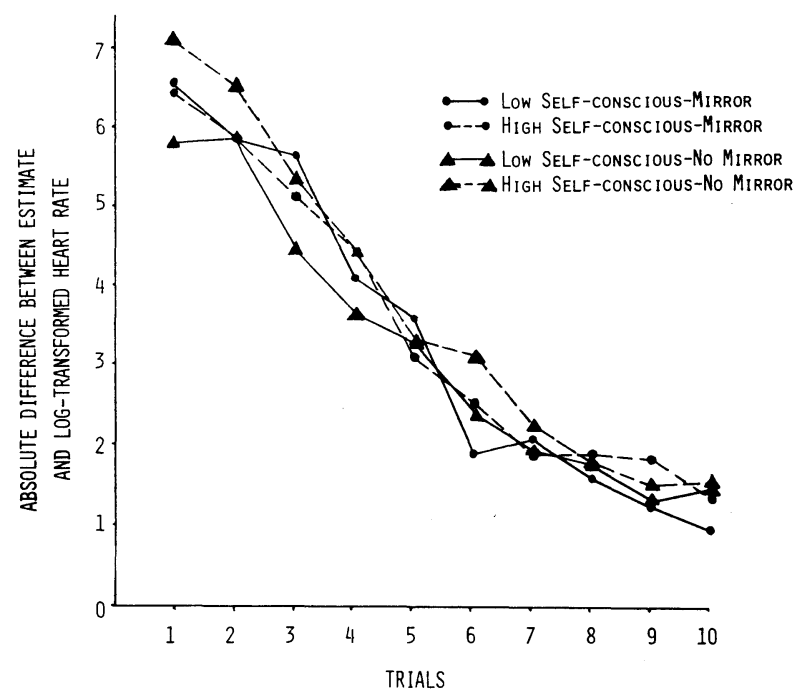

Figure 2. Absolute differences between estimates and logtransformed standardized heart rates, over 1030 -sec intervals, among persons low and high in self-consciousness, with mirror present and mirror absent. 
Transformed scores. It is generally accepted, however, that perceived stimulus intensity increases as a logarithmic function of the actual intensity of the stimulus being estimated (e.g., Stevens, 1962). It seems reasonable that this principle would apply to internal, as well as external, perceptual events. Therefore, each subject's standardized heart rate scores were submitted to a natural $\log$ transformation, difference scores were computed between these values and the subject's estimates, and the absolute values of these difference scores were submitted to another repeated-measures analysis of variance. Again, however, only a significant trials effect emerged $[F(9,432)=134.99, p<.001]$ (see Figure 2).

\section{DISCUSSION}

It had been predicted that heightened self-focus, either situationally manipulated or dispositional, would result in enhanced accuracy of heart rate estimation. Greater accuracy should have been reflected in smaller differences between actual and estimated heart rate. The data from this study offered no support for this prediction, however, in either of two analyses.

There are at least three possible explanations for this failure to confirm our predictions. One possibility is that self-focus does not really lead to enhanced awareness of internal states. We regard this possibility as the least plausible of the three because (1) self-attention has been reliably associated with exaggerated reports of emotional states on at least six occasions (Carver et al., 1979; Scheier, 1976; Scheier \& Carver, Experiments 14), and (2) self-attention has been associated with greater accuracy in the face of misleading information on at least three occasions (Gibbons et al., 1979; Scheier et al., 1979, Experiments 1 and 2).

A second possibility stems from a closer examination of these previous findings. Specifically, it may be that our predictions in the present study were misguided. That is, previous findings indicate that self-focus led to exaggerated reports of emotional experience, when emotion was present. Perhaps we should have expected subjects who were experiencing heart rate elevations to report greater elevation when self-focus was high than when it was lower. Similarly, the previous demonstrations that self-focus leads to greater accuracy did so in the presence of misleading information. It is quite plausible that, in the absence of such misdirection attempts, no such enhancement of accuracy occurs. In effect, what this reasoning would predict is no difference in self-reports as a function of self-focus when internal stimuli are near baseline levels (and no misdirection is attempted) and a gradually increasing difference in self-report intensity as the veridical state departs from baseline.

A reexamination of the data of this study provides some suggestive evidence of the occurrence of such a process, at least as a function of dispositional self-consciousness. Of greatest relevance are data from subjects in no-mirror conditions, because of the fact that mirror presence often minimizes differences due to dispositional self-consciousness (cf. Carver \& Scheier, 1978). Examination of Figures 1 and 2 indicates greater initial errors by persons high in self-consciousness than by those lower in self-consciousness. Inasmuch as virtually all errors at this stage were overestimations, this means that highly self-conscious persons were reporting greater heart rate elevations than less self-conscious persons. This initial difference was statistically reliable, both when computed on untransformed data $[\mathrm{t}(26)=$ $2.88, \mathrm{p}<.01]^{2}$ and when computed on transformed data $[\mathrm{t}(26)=2.20, \mathrm{p}<.05]$. To argue that this reflects the process postulated above, however, raises the question of why there was no comparable effect for mirror presence. Our answer is speculative: Perhaps awareness of one's heart rate depends on greater amounts of experience than were provided in this study. A self-consciousness difference might have emerged only because this is a long-term personality disposition, which presumably has operated over many years. Temporarily enhancing self-focus by mirror presence, on the other hand, may not have enabled subjects to gain sufficient access to their heart rates to affect their ratings.

The latter notion brings us to a third possibility. Perhaps heart rate is simply a very poor "channel" for persons to monitor. It may be more difficult to gain subjective access to heart rate information than to other sorts of information, such as muscle tension or blood pressure. This would be consistent with our speculation (above) as to why dispositional selfconsciousness might have a more predictable effect here than manipulated self-attention. Suggestive support for this possibility comes from research on subjects with electronic pacemakers (e.g., Nowlin, Eisdorfer, Whalen, \& Troyer, 1971). This research found it to be possible to create paced increases in subjects' heart rates of up to $110 \mathrm{bpm}$, without their awareness. It seems noteworthy, in this regard, that previous research (e.g., Gibbons et al., 1979; Scheier \& Carver, 1977) has asked subjects to report rather "undifferentiated" arousal. Perhaps, given such freedom, previous subjects gravitated to those informational channels that are most easily monitored.

At present, these various possibilities must be treated as equally plausible. To determine which of them is the more accurate will require more study.

\section{REFERENCES}

Carver, C. S., Blaney, P. H., \& Scheier, M. F. Focus of attention, chronic expectancy, and responses to a feared stimulus. Journal of Personality and Social Psychology, 1979, 37, 1186-1195.

Carver, C. S., \& Scheier, M. F. Self-focusing effects of dispositional self-consciousness, mirror presence, and audience presence. Journal of Personality and Social Psychology, 1978, 36, 324-332.

Fenigstein, A., Scheier, M. F., \& Buss, A. H. Public and private self-consciousness: Assessment and theory. Journal of Consulting and Clinical Psychology, 1975, 43, 522-527.

Gibbons, F. X., Carver, C. S., Scheier, M. F., \& Hormuth, S. Self-focused attention and the placebo effect: Fooling some of the people some of the time. Journal of Experimental Social Psychology, 1979, 15, 263-274.

Nowlin, J. B., Eisdorfer, C., Whalen, R., \& Troyer, W. G. The effect of exogenous changes in heart rate and rhythm upon reaction time performance. Psychophysiology, 1971, 7, 186-193.

Scheier, M. F. Self-awareness, self-consciousness, and angry aggression. Journal of Personality, 1976, 44, 627-644.

Scheier, M. F., \& Carver, C. S. Self-focused attention and the experience of emotion: Attraction, repulsion, elation, and depression. Journal of Personality and Social Psychology, 1977, 35, 625-636.

Scheier, M. F., Carver, C. S., \& Gibbons, F. X. Self-directed attention awareness of bodily states, and suggestibility. Journal of Personality and Social Psychology, 1979, 37, 1576-1588.

Stevens, S. S. The surprising simplicity of sensory metrics. American Psychologist, 1962, 17, 29-39.

\section{NOTES}

1. This instrument has two separate subscales. Private selfconsciousness reflects awareness of covert or hidden aspects of oneself (e.g., thoughts, feelings, and motives). Public selfconsciousness reflects awareness of social self-aspects. It clearly is the private dimension that is relevant to the present research.

2. Each of these comparisons was computed independently, using the variances of the relevant cell means.

(Received for publication December 5, 1979.) 